\title{
Phonon modes and heat capacity of monolayer films adsorbed in spherical pores
}

\author{
R. A. Trasca, ${ }^{1}$ A. M. Vidales,${ }^{2}$ and M. W. Cole $^{1}$ \\ ${ }^{1}$ Department of Physics, Pennsylvania State University, University Park, Pennsylvania 16802 \\ and Materials Research Institute, University Park, Pennsylvania 16802 \\ ${ }^{2}$ Departamento de Fisica y CONICET, Universidad Nacional de San Luis, 5700 San Luis, Argentina
}

(Received 8 November 2002; published 11 March 2003)

\begin{abstract}
We examine the hydrodynamic phonon spectrum of a monolayer film adsorbed on the wall of a spherical pore. Due to the boundary conditions, the monolayer film exhibits a discrete phonon spectrum. The corresponding density of states per unit frequency is thus a set of $\delta$ functions, and the heat capacity exhibits Arrhenius behavior at low temperatures. At high temperatures, the heat capacity approaches the $\mathrm{C} \sim T^{2}$ behavior of a two-dimensional monolayer film. Our results for the spherical surface film are compared to previous calculations for films confined to a cylindrical surface.
\end{abstract}

DOI: 10.1103/PhysRevE.67.031602

PACS number(s): 68.15.+e

Monolayer films have been extensively studied, both theoretically and experimentally, in part because they exhibit reduced dimensionality and novel phases $[1,2]$. For example, low pressure adsorption on graphite usually yields a twodimensional (2D) monolayer, whereas adsorption in carbon nanotubes can result in quasi-one-dimensional behavior [3-6]. As a result of the reduced dimensionality, the phonon spectrum and the thermodynamic properties of such films are altered with respect to bulk properties. The basic reason is that the substrate imposes its boundary conditions on the adsorbate. For example, previous calculations of Vidales, Crespi, and Cole (VCC henceforth) show that the heat capacity of a monolayer film adsorbed on the inner wall of a carbon nanotube exhibits a crossover from quasi-onedimensional to two-dimensional behavior when the thermal phonon wavelength $\lambda=\hbar s \beta$ approaches $2 \pi R$, the circumference of the cylindrical adsorbed film ( $s$ is the sound speed and $\beta^{-1}=k_{B} T$ ) [7]. Due to the cylindrical geometry, finite in the radial direction and (assumed) infinite in the axial direction, the surface-parallel phonons have a dispersion relation:

$$
\omega_{n k}=s \sqrt{k^{2}+(n / R)^{2}},
$$

where $k$ is the quasicontinuous wave vector (parallel to the cylinder axis) and $n$ is the azimuthal quantum number.

Here we consider the analogous case of a thin film adsorbed on the inner surface of a spherical cavity. The outline of this paper is as follows. We derive the in-plane phonon dispersion relation for the spherical surface film and compare the density of states with corresponding results for the cylindrical and planar cases. This leads us to anticipate some of the heat capacity results. Finally, we identify a crossover behavior in the heat capacity and compare this with some experimental measurements.

In the derivation of the phonon dispersion relation, we adopt a method similar to that of the VCC, concerned with cylindrical films [7]. It is noted first that at low $T$, the surface-normal vibrations can be ignored since they acquire a gap at roughly the first surface-normal excitation energy of the isolated atom ( $~ 60 \mathrm{~K}$ for ${ }^{4} \mathrm{He}$ on graphite). Thus, in a liquid monolayer film, only the surface-parallel phonons are excited at low $T$. These are described by a continuous two- dimensional wave vector $k$ for a planar film and a onedimensional wave vector in the cylindrical case. Since a spherical film is confined (effectively zero dimensional), we expect the quantum number equivalent to a wave vector $k$ to have discrete values. The derivation of the spectrum follows from the scalar Helmholtz equation, describing the velocity potential $\Psi$ :

$$
\nabla^{2} \Psi+k^{2} \Psi=0
$$

where $k \equiv \omega / s$. This yields solutions of the form $\Psi(\cos \theta, \phi)=P_{l}^{m}(\cos \theta) \Phi(\phi)$ [where $\Phi(\phi)=e^{i m \phi}, m=0$, \pm 1 , $\pm 2, \ldots$; and $P_{l}^{m}$ are the associated Legendre polynomials for integers $l \geqslant|m|]$. The boundary condition imposed on the Legendre polynomials (to be finite when $\cos \theta= \pm 1$ ) yields the expected discreteness of the eigenvalue $k=\sqrt{l(l+1)} / R$. The dispersion relation for the spherical film follows as

$$
\omega_{l}=\sqrt{l(l+1)}(s / R)
$$

The corresponding density of states per unit frequency, $g(\omega)$, is thus given by a sum of $\delta$ functions, with multiplicity given by the $m$ degeneracy $(2 l+1)$ of each energy level:

$$
g(\omega)=\sum_{l} g_{l}^{s p h}(\omega)=\sum_{l}(2 l+1) \delta\left(\omega-\omega_{l}\right)
$$

We want to compare the spherical film results with the cylindrical and planar densities of states. For the cylindrical film, the number of states within the interval $d \omega$ is

$$
g^{c y l}(\omega) d \omega=2 \frac{L}{2 \pi} d k,
$$

where $L /(2 \pi)$ is the number of states in the $1 \mathrm{D}$ reciprocal space range $d k$, and the factor 2 arises from the $( \pm k)$ degeneracy. Using the dispersion relation [Eq. (1)], the cylindrical density of states corresponding to a specific band $n$ is

$$
g_{n}^{c y l}(\omega)=\frac{L}{\pi s} \frac{\Theta(\omega-s|n| / R)}{\sqrt{1-(s n / \omega R)^{2}}},
$$




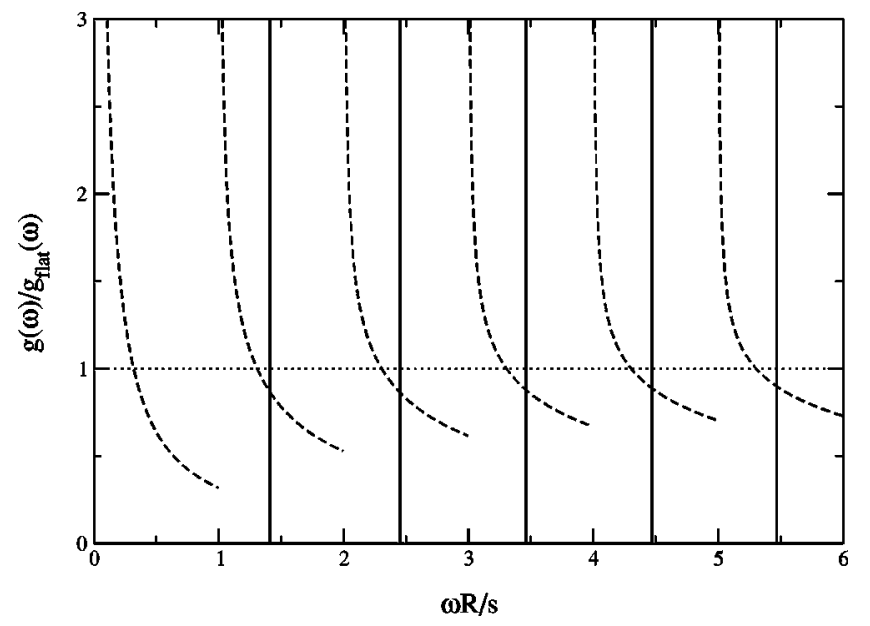

FIG. 1. The density of states of the cylindrical film (dashed curve) relative to that of planar film (dotted line) as a function of the reduced frequency $\omega R / s$. The full vertical lines indicate the delta functions $g_{s p h}(\omega)$ for the spherical film.

where $\Theta(x)$ is the Heaviside step function ( 1 for $x \geqslant 0$ and 0 for $x<0$ ). The total density of states is the sum over all bands:

$$
g^{c y l}(\omega)=\sum_{n=-\infty}^{\infty} g_{n}^{c y l}(\omega)
$$

At low $T$, the azimuthal excitations $(n \geqslant 1)$ are frozen out due to the gap $E_{10}=\hbar s / R$ (see Ref. [7]). Thus, the dispersion relation [Eq. (1)] becomes $\omega=s k$ when $n=0$, and the cylindrical density of states becomes constant $g_{0}(\omega)=L / \pi s$. The planar film has also a linear dispersion relation $\omega=s k$, but $k$ is then a $2 \mathrm{D}$ wave vector. The corresponding density of states is

$$
g^{2 D}(\omega)=\frac{A}{2 \pi} \frac{\omega}{s^{2}}
$$

where $A$ is the area of the surface.

The spherical, cylindrical, and planar densities of states are displayed in Fig. 1. In order to get dimensionless numbers, we plot the density of states relative to the planar case for the same area $(A=2 \pi R L$ in the cylindrical case and $A$ $=4 \pi R^{2}$ in the spherical case). Notice that the largest density of states at low $\omega$ corresponds to the cylindrical film, followed by the planar film, and finally, by the spherical film. This implies that the cylindrical film will exhibit the largest heat capacity (per unit area) at low $T$, followed by the planar and then the spherical film. At high $\omega$, however, the number of states in an interval $d \omega$ is the same for all types of films considered, as shown in the Appendix. Therefore, the heat capacities of the spherical, planar, and cylindrical films should approach asymptotically the same values at high $T$. We note that the "high $T$ " limit in this elastic regime differs from the very high $T$ limit in an experiment. That is, the law of Dulong and Petit corresponds to a very high $T$ constant behavior of $C$. The crossover to the latter regime occurs when $k T$ exceeds the Debye temperature. We do not consider this regime of $T$ here.

We turn our attention to the thermodynamic properties of the spherical film. Using Eq. (3), the energy of a phonon is

$$
E_{l}=\frac{\hbar s}{R} \sqrt{l(l+1)} \text {. }
$$

There is no mode for $l=0$, and thus the spherical film possesses a gap $E_{1}=(\hbar s \sqrt{2}) / R$. The total energy of the phonon system is

$$
E_{s p h}=\sum_{l=1,2, \ldots} \frac{(2 l+1) E_{l}}{\exp \left(\beta E_{l}\right)-1}
$$

where $2 l+1$ is the degeneracy of the energy levels. In the high $T$ limit, the sum can be replaced by an integral, which can be evaluated analytically (because $1 \ll l$ for the predominant modes):

$$
E_{s p h} \sim \frac{\hbar s}{R} \int_{0}^{\infty} \frac{2 l^{2} d l}{\exp (\beta \hbar s l / R)-1}=\frac{A \zeta(3)}{\pi \beta^{3} \hbar^{2} s^{2}},
$$

where $A=4 \pi R^{2}$ is the film area and $\zeta$ is the Riemann zeta function. As in the case of the cylindrical film, the high $T$ heat capacity per unit area of the spherical film is the same as that of a planar film:

$$
\frac{C_{2 D}(T)}{k_{B} A}=\frac{3 \zeta(3)}{\pi \beta^{2} \hbar^{2} s^{2}} .
$$

For simplicity, we define a reduced temperature

$$
t=R /(\beta \hbar s),
$$

and the dimensionless heat capacity takes the form

$$
C_{s p h} / k_{B}=t^{-2} \sum_{l=0,1, \ldots} \frac{l(l+1)(2 l+1) e^{\sqrt{l(l+1)} / t}}{\left(e^{\sqrt{l(l+1)} / t}-1\right)^{2}} .
$$

As in the case of the cylindrical film [7], the heat capacity is seen to be a universal function of the reduced temperature [the right side of Eq. (13)]. Figure 2 shows the dependence on $T$ of the spherical and cylindrical heat capacities, compared to the planar heat capacity $\left(C / C_{\text {planar }}\right)$. Notice that the planar film is the high $T$ limit for both the spherical and cylindrical films, as expected. However, the heat capacity of the cylindrical film approaches it from above, whereas the heat capacity of the spherical film approaches it from below. This behavior was expected from the previous discussion of the densities of states. We note that the asymptotic approach to the planar limit occurs at a lower $T$ in the cylindrical case than in the spherical case. This can be explained in at least two ways. One is that a cylinder (having one infinite radius of curvature) is closer to a plane than a sphere is. The other way is that the density of states (Fig. 1) of the cylinder is closer to that of a planar surface than is the density of states for the sphere. In analogy to the crossover behavior of the 


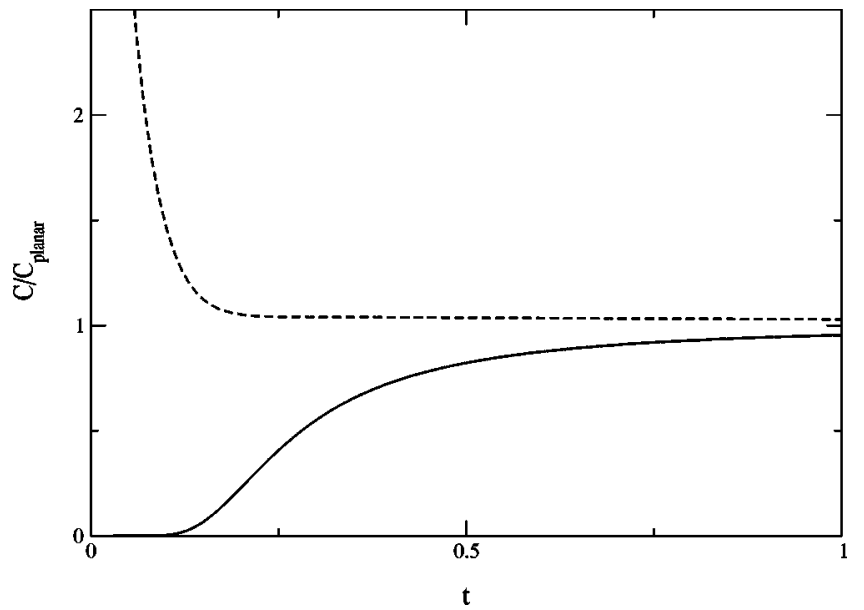

FIG. 2. The heat capacity of a cylindrical (dashed curve) and a spherical (full curve) film relative to that of a planar film, as a function of the reduced temperature defined in Eq. (13).

cylindrical film [7], one may speak of a crossover temperature $T_{\text {cross }}$ from a zero-dimensional system to a twodimensional system, defined as the temperature where the heat capacity is about half of that of a planar film. In the present case, from Fig. $2, k_{B} T_{\text {cross }} \sim \hbar s / 4 R$. At low $T$, the spherical heat capacity has an activated behavior:

$$
C_{s p h} / k_{B}=6 t^{-2} \exp (-\sqrt{2} / t) .
$$

We do not know of any experiments related to adsorption in spherical pores, but the irregular confined pores of a disordered material may conceivably be approximated as spherical pores. Crowell and Reppy have investigated ${ }^{4} \mathrm{He}$ films adsorbed in two porous glasses, Vycor and aerogel, using high-precision torsional oscillator and calorimetry techniques [8-10]. At some critical coverage, the ${ }^{4} \mathrm{He}$ film undergoes a transition from nonsuperfluid to superfluid behavior. In the nonsuperfluid regime, the heat capacity exhibits a linear dependence at large $T$, but $C / T$ drops rapidly at low $T$, so a crossover temperature between these two behaviors is defined and calculated to be about $100 \mathrm{mK}$ for the lowest coverage. In the context of our calculations, a working hypothesis is that the pore geometry is like an American football, i.e., an ellipse of revolution. This could show the behavior of a cylinder $(C \sim T)$ at modest $T$, but exhibits a gap at low $T$, as in the case of a spherical geometry. We may estimate the heat capacity at low $T$ with our spherical pore model, and use the cylindrical pore for higher $T$. For a pore diameter of about $70 \AA$, as in the case of Vycor pores, we assume that the ${ }^{4} \mathrm{He}$ atoms form a spherical film of about 60 $\AA$ diameter, so that there are about $10^{3} \mathrm{He}$ atoms in a pore. Then the heat capacity per mole is of the order of $\mathrm{mJ} / \mathrm{K}$, the same order of magnitude as the experimental heat capacity of the nonsuperfluid ${ }^{4} \mathrm{He}$ film. However, the calculated heat capacity exhibits the characteristic activated behavior seen in Eq. (15), and does not follow the general trend of the experimental measurements, which looks more like a power law behavior. Also, the heat capacity experimental values at higher $T$ can be obtained with our cylindrical pore model, but only with an unreasonably large speed of sound. However, the crossover temperature given by our spherical model is of the order of $150 \mathrm{mK}$, comparable to the experimental crossover of $100 \mathrm{mK}$. Thus, it may happen that the geometry of the Vycor pores produces boundary conditions for the film, whose effect changes with increasing temperature, leading to a crossover behavior.

In conclusion, the substrate geometry imposes constraints on the phonons of monolayer films adsorbed within a spherical pore. The first effect of the confinement is the discreteness of the phonon spectrum. Because of a low $\omega$ gap in the spectrum, the specific heat exhibits an essential singularity at $T=0$, whereas at high $T$, it asymptotes to that of a planar film $\left(C \sim T^{2}\right)$. A somewhat different crossover behavior occurs in the case of a cylindrical film, which has quasi-onedimensional behavior at low $T(C \sim T)$ and planar (2D) behavior at high $T$. This pair of solutions may explain some of the heat capacity features of monolayers adsorbed in disordered porous materials, whose various pores have different geometries and sizes.

We are grateful to the National Science Foundation, which has supported this research. We thank Paul Crowell and Moses Chan for helpful communications.

\section{APPENDIX}

This paper states that the density of states at high $\omega$ is the same for all three problems discussed here. We will give now a proof of this statement. Let us start with the case of a spherical film, and choose an interval $d \omega$ between $\omega_{1}$ $=\sqrt{l(l+1)}(s / R)$ and $\omega_{2}=\sqrt{(l+1)(l+2)}(s / R)$, corresponding to consecutive values of 1 , when $l \gg 1$. The number of states in this interval is

$$
\int_{\omega_{1}}^{\omega_{2}} g^{s p h}(\omega) d \omega=(2 l+1) \int_{\omega_{1}}^{\omega_{2}} \delta\left(\omega-\omega_{l}\right)=2 l+1 .
$$

Thus, in the limit of high $\omega$, and correspondingly high $l$, the number of states in an interval $d \omega$ is of the order $2 l$.

For the planar film, we consider the same interval $d \omega$, and the number of states in this interval is

$$
\int_{\omega_{1}}^{\omega_{2}} \frac{A}{2 \pi s^{2}} \omega d \omega=2(l+1),
$$

where the area of the planar film equals the area of the spherical film, $A=4 \pi R^{2}$. For high 1 , the number of states of the planar film in this interval $d \omega$ is therefore of the order of $2 l$, equal to that of the spherical film.

The last case is that of a cylindrical film. The number of states corresponding to the interval $d \omega$ is 


$$
\begin{aligned}
& \int_{\omega_{1}}^{\omega_{2}} \frac{L}{\pi s}\left(\sum_{n=-l}^{l} \frac{\Theta(\omega-s n / R)}{\sqrt{1-(s n / \omega R)^{2}}}\right) d \omega \\
& =\frac{L}{\pi s} \sum_{n=-l}^{l} \int_{\omega_{1}}^{\omega_{2}} \frac{d \omega}{\sqrt{1-s n / \omega R)^{2}}} \\
& =\frac{L}{\pi R} \sum_{n=-l}^{l}\left(\sqrt{(l+1)(l+2)-n^{2}}-\sqrt{l(l+1)-n^{2}}\right) .
\end{aligned}
$$

First, in order to compare the cylindrical and spherical films, we take their areas to be the same; thus $2 \pi R L=4 \pi R^{2}$ or
$L=2 R$. Then, if we define $\sqrt{l(l+1)-n^{2}}$ as $f_{n}(l)$, a function of variable 1 , the result of integration can be written as $f_{n}(l$ $+1)-f_{n}(l)$, which (for large 1$)$ is the derivative $f_{n}^{\prime}(l)$. In the limit of large $1, f_{n}(l) \sim \sqrt{l^{2}-n^{2}}$, whose derivative is $l / \sqrt{l^{2}-n^{2}}$. Converting the sum to an integral, the number of states becomes

$$
N_{\text {states }}=\frac{2}{\pi} \sum_{n=-l}^{l} \frac{l}{\sqrt{l^{2}-n^{2}}} \simeq \frac{2}{\pi} l \int_{-l}^{l} \frac{d n}{\sqrt{l^{2}-n^{2}}}=2 l .
$$

Therefore, at high frequency, the integrated number of states in the interval $d \omega$ is the same in all three cases.
[1] M.M. Calbi, M.W. Cole, S.M. Gatica, M.J. Bojan, and G. Stan, Rev. Mod. Phys. 73, 857 (2001).

[2] L.W. Bruch, M.W. Cole, and E. Zaremba, Physical Adsorption: Forces and Phenomena (Oxford University Press, Oxford, 1997).

[3] M. Boninsegni, S.Y. Lee, and V.H. Crespi, Phys. Rev. Lett. 86, 3360 (2001).

[4] M.W. Cole, V.H. Crespi, G. Stan, C. Ebner, J.M. Hartman, S. Moroni, and M. Boninsegni, Phys. Rev. Lett. 84, 3883 (2000).

[5] M.C. Gordillo, J. Boronat, J. Casulleras, Phys. Rev. B 61, R878 (2000).
[6] W. Teizer, R.B. Hallock, and E. Dujardin, Phys. Rev. Lett. 84, 1844 (2000).

[7] A.M. Vidales, V.H. Crespi, and M.W. Cole, Phys. Rev. B 58, 13426 (1998).

[8] P.A. Crowell, F.W. Van Keuls, and J.D. Reppy, Phys. Rev. B 55, 12620 (1997).

[9] P.A. Crowell and J.D. Reppy, S. Mukherjee, J. Ma, M.H.W. Chan, and D.W. Schaefer, Phys. Rev. B 51, 12721 (1995).

[10] P.A. Crowell, F.W. Van Keuls, and J.D. Reppy, Phys. Rev. Lett. 75, 1106 (1995). 